\title{
Certain Aspects of the Gravitational Field of a Disk
}

\section{Jonathan D. Weiss}

JSA Photonics, LLC, Albuquerque, NM, USA

Email: Jondweiss@aol.com

How to cite this paper: Weiss, J.D. (2018) Certain Aspects of the Gravitational Field of a Disk. Applied Mathematics, 9, 13601377.

https://doi.org/10.4236/am.2018.912089

Received: November 26, 2018 Accepted: December 24, 2018

Published: December 27, 2018

Copyright $\odot 2018$ by author and Scientific Research Publishing Inc. This work is licensed under the Creative Commons Attribution International License (CC BY 4.0).

http://creativecommons.org/licenses/by/4.0/

\begin{abstract}
There are at least two reasons why one would study the gravitational field of a disk. The first is that many astronomical objects, such as spiral galaxies like the Milky Way, are disk-like. The second is that the field of a disk is interesting, particularly when compared to that of a spherical, or near-spherical, object, which is much easier to analyze because of its high degree of symmetry. It is hoped that this study will augment previous work on this subject. The aspects presented in this paper are as follows: 1) both the radial and vertical gravitational fields of a thin disk within the plane of the disk and above it; 2) a comparison of some of the field results obtained by Lass and Blitzer (1983) involving elliptic integrals to those obtained by a standard numerical integration, now available online, and separately through the use of Legendre polynomials; 3 ) the logarithmic divergence of the radial field at the edge of a thin disk; 4) the fields in the plane of a disk containing a central hole, particularly within the hole, such as the rings of Saturn; 5) circular orbits within the plane of a single disk and half way between two disks, and their stability; 6) the escape velocity at a point within the Milky Way, particularly at the position of the solar system and without any added, or subtracted, orbital effects around the galactic center; and 7) the radial field at the circular edge of a disk of finite thickness.
\end{abstract}

\section{Keywords}

Disk-Shaped Mass, Gravitational Effects of a Disk-Shaped Mass, Calculational Techniques of Such Gravitational Effects

\section{Introduction}

Although many astronomical objects, such as stars, planets, and certain galaxies, are spherical, or nearly spherical, in shape, many others are disk-like. Examples 
of the latter are bar spiral galaxies (such as the Milky Way), the rings of Saturn, viewed as disks with a large central hole, and protoplanetary disks, consisting of dense gas and dust surrounding a newly formed star that add mass to the star [1]. Thus, the gravitational fields of such structures have been studied, and we hope that the work presented here on various aspects of the gravitational fields of uniform disks, albeit a geometric simplification, will augment previous work and be of general interest as a problem in physics.

\section{Certain Previous Work (Lass and Blitzer)}

Two examples of previous work on the gravitational field of an infinitely thin disk are by Lass and Blitzer [2] and Krough, Ng, \& Snyder [3], both published in the early 1980s. Neither presented specific graphic examples of their mathematical results, which would have made them more concrete for the reader. We will do so here, but only for the paper by Lass and Blitzer. Other augmentations of the paper by Lass and Blitzer will also presented.

With reference to Figure 1, the following is their expression for the gravitational potential, $V(r, z)$, for $r<a$.

$$
\begin{aligned}
V(r, z)= & 2 \sigma G\left[\pi|z|-\sqrt{z^{2}+(a+r)^{2}} E(k)\right. \\
& -\left[\left(a^{2}-r^{2}\right) / \sqrt{z^{2}+(a+r)^{2}}\right] K(k) \\
& \left.-\left[((a-r) /(a+r)) z^{2} / \sqrt{z^{2}+(a+r)^{2}}\right] \Pi(n, k)\right] .
\end{aligned}
$$

For $r>a$, the first term, $\pi|z|$, is eliminated because there is no surface mass beyond $r=a$. Thus, the $\mathrm{z}$-component of the field at $z=0$ is zero there, while it is not zero for $z<a$, where there is surface mass. Regarding the meaning of the symbols not noted in Figure 1, $\sigma$ is the mass per unit area of the disk; $G$ is Newton's gravitational constant, and $K(k), E(k)$, and $\Pi(n, k)$ are the complete elliptic integrals of the first, second, and third kind, respectively. The parameters $\mathrm{k}$ and $n$ are defined such that $k^{2}=4 a r /\left[z^{2}+(a+r)^{2}\right]$ and $n=4 a r /(a+r)^{2}$. We note that at $z=0, k^{2}=n$. As to the complete elliptic integrals, they have the following integral representations, as stated in [4],

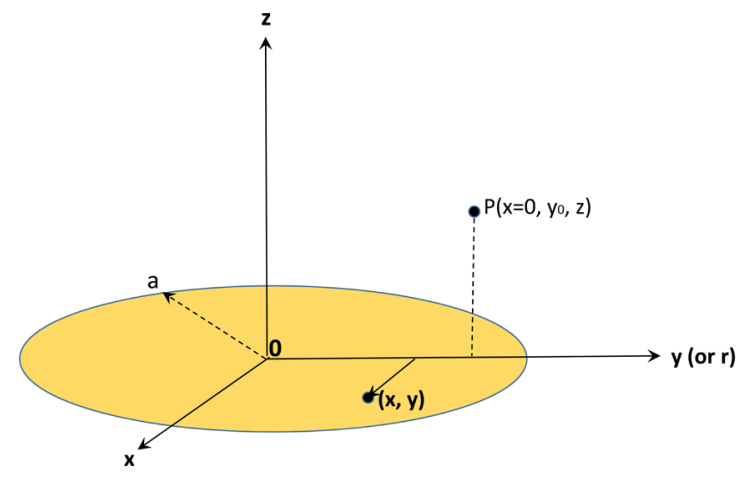

Figure 1. Basic geometry; uniform circular gravitational disk of radius a. $P\left(x_{0}, y_{0}, z\right)$ is the field point and $(x, y)$ is the position of a mass point on the disk surface. The $y$-axis can be considered $\mathrm{r}$ because of the azimuthal symmetry of the disk. 


$$
\begin{aligned}
& K(k)=\int_{0}^{1} \frac{\mathrm{d} t}{\sqrt{\left(1-t^{2}\right)\left(1-k^{2} t^{2}\right)}} ; \\
& E(k)=\int_{0}^{1} \frac{\mathrm{d} t \sqrt{1-k^{2} t}}{\sqrt{1-t^{2}}} ; \\
& \Pi(n, k)=\int_{0}^{1} \frac{\mathrm{d} t}{\left(1-n t^{2}\right) \sqrt{\left(1-t^{2}\right)\left(1-k^{2} t^{2}\right)}} ;
\end{aligned}
$$

Although it is of no consequence, we should be aware of a slight difference in notation between [2] and [4] What [4] refers to as $n$ is called $n^{2}$ in [2]. Since [4] is a more modern publication, we shall adhere to its convention and define $4 a r /(a+r)^{2}$ as $n$.

To obtain the force in the radial direction, $F_{r}$, we calculate $-\partial V(r, z) / \partial r$, which involves the sums of the derivatives of the three products of functions in Equation (1). When that is done, the force within the plane of the disk (where $z=0$ ) can be expressed as:

$$
\begin{aligned}
& F_{r}(z=0) / 2 G \sigma \\
& =-\left[\frac{1+r_{a}^{2}}{r_{a}\left(1+r_{a}\right)}\right] K(k)+\left[\frac{\left(1+r_{a}\right)\left(2+r_{a}\right)}{2 r_{a}}\right] E(k)-\left[\frac{\left(1-r_{a}\right)^{2}}{2\left(1+r_{a}\right)}\right] \Pi(n, k)
\end{aligned}
$$

In this equation, $r_{a} \equiv r / a$, is a relative coordinate. If $z \neq 0$, then $z_{a} \equiv z / a$, would also be used in the equation for $F_{r}(z \neq 0) / 2 G \sigma$. Since the product rule for derivatives is used in deriving Equation (3), one might wonder why no derivatives of the elliptic integrals appear in it. This apparent anomaly exists because the derivatives of all three elliptic integrals can be expressed in terms of the elliptic integrals themselves, as shown in [4].

The scaled radial field in the plane of the disk is plotted in Figure 2, for $0 \leq r_{a}$ $\leq 1$. The elliptic integrals were evaluated numerically with the help of "Keisan Online Calculator" [5]. The field is negative because it is directed in the negative r-direction. For comparison, the scaled radial field for a solid sphere of radius " $a$ " is shown in Figure 3. The two fields are qualitatively similar, but the field for a sphere increases linearly in $r_{a}$ for $r_{a} \leq 1$ and is finite for $r_{a}=1$. That is not true for the field of the disk, a two-dimensional object, for which the field behavior is curved for $r_{a}<1$ and diverges to negative infinity at $r_{a}=1$, as suggested by the arrow pointing straight down at that point in Figure 2. The behavior of the curve near $r_{a}=1$ is logarithmic, and is caused by $K(k)$ in that region. The logarithmic behavior of $K(k)$ for $0.8 \leq r_{a} \leq 1.2$ is shown in Figure 4, although that graph is a specific numerical evaluation, as opposed to an analytic proof. However, an analytic proof is presented in "Mathematics Stack Exchange" [7] for elliptic integrals. The logarithmic divergence at $r_{a}=1$ is reasonable when one considers the divergence at a point mass (of zero dimension) to be $\left(1 / r^{2}\right)$ and a line mass of any length to be a more gradual (1/r) (one-dimensional). In this case, we are dealing with the edge of a two-dimensional mass distribution and expect an even more gradual divergence, which the logarithmic divergence is. 


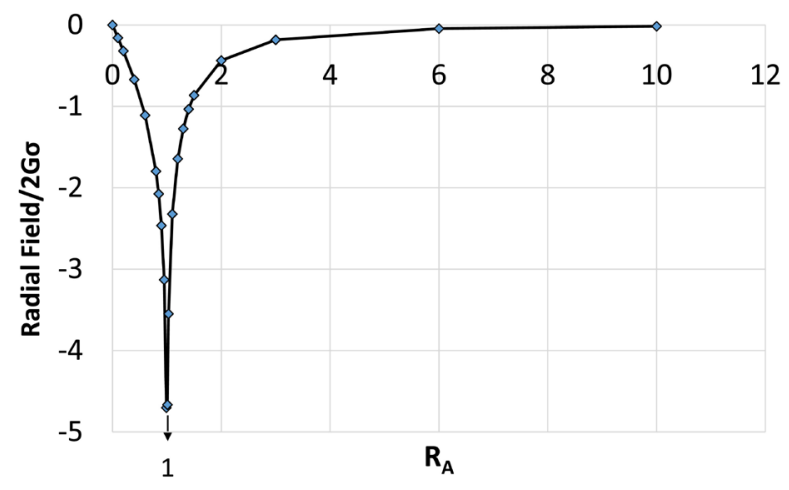

Figure 2. Radial gravitational field derived from [2]. Downward arrow indicates logarithmic divergence to negative infinity exactly at $r_{a}=1$, caused by the complete elliptic integral of the first kind at $k=1, K(1)$.

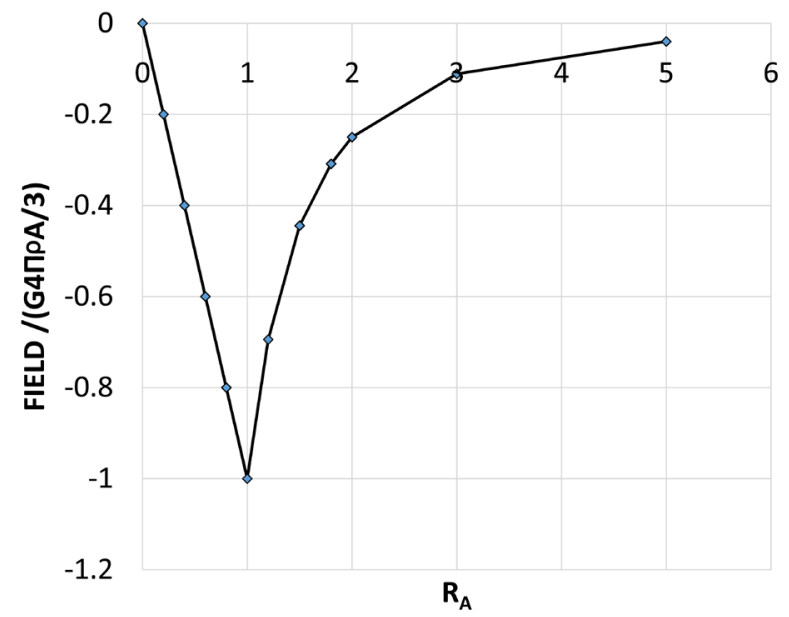

Figure 3. Gravitational field of a solid sphere.

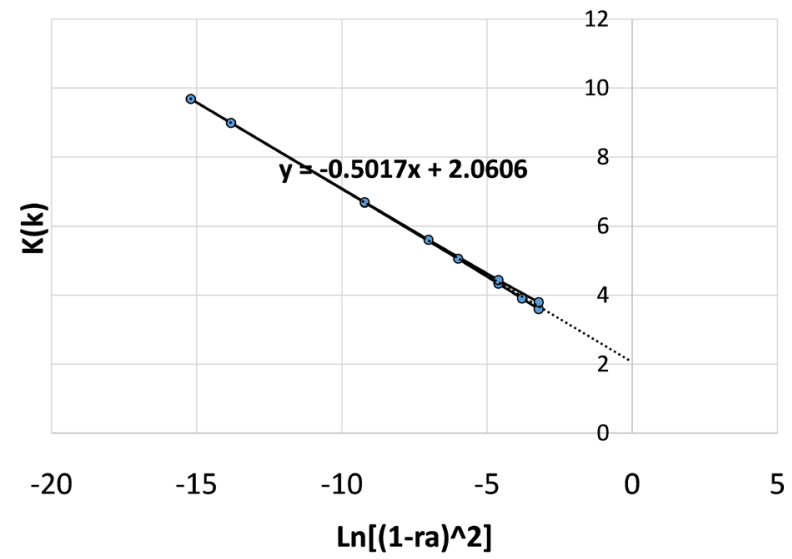

Figure 4. Linear-Ln plot of $K(k)$ from $0.8 \leq r_{a} \leq 1.2$. The shape of the curve indicates that $K(k)$ varies logarithmically with $\left|1-r_{a}\right|$ near $r_{a}=1$ and diverges at that point.

In addition, a view of the complete elliptic integral of the third kind in [5], when both arguments are near unity, suggests a divergence of $\Pi(n, k)$ in that region. Indeed, it does diverge, but with what behavior? The graph in Figure 5, 


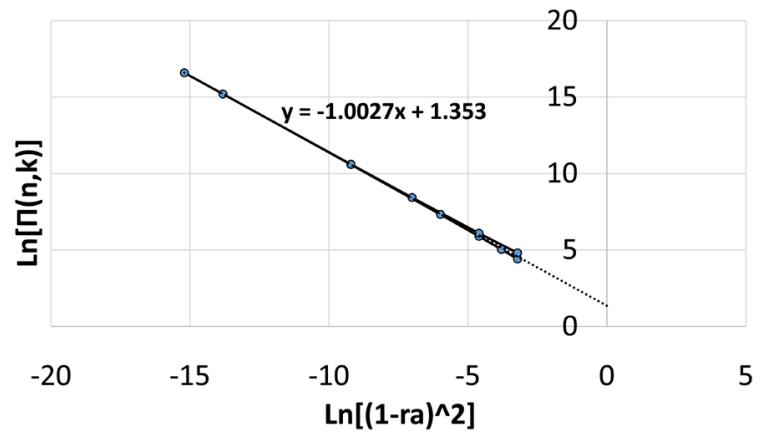

Figure 5. n-Ln plot of $\Pi(n, k)$ vs $\left(1-r_{a}\right)^{2}$ for $0.8 \leq r_{a} \leq 1.2$, showing that the logarithms of these two functions are linearly related with a slope of -1 . This relationship demonstrates that $\Pi(n, k)$ varies as $1 /\left[\left(1-r_{a}\right)^{2}\right]$ in this region near $r_{a}=1$.

obtained in the same way as that for $K(k)$, demonstrates that $\Pi(n, k)$ diverges as $1 /\left(1-r_{a}\right)^{2}$. However, its coefficient in Equation (3) contains $\left(1-r_{a}\right)^{2}$. Thus, the final term in Equation (3) is finite at $r_{a}=1$, as is the second term because of the normal behavior of $E(k)$ over the entire range of $\mathrm{k}$. Therefore, the behavior of the field at $r_{a}=1$ is due do solely to $K(k)$ there.

\section{Certain Current Work}

In this section, we examine two other methods of calculating the same radial field as above. These are the use of "standard" numerical integration and Legendre Polynomials.

\section{Numerical Integration}

Figure 6 is relevant to the use of numerical integration.

The gravitational field, $V\left(r_{o}\right)$, will be first calculated and then $F_{r}\left(r_{o}\right)$ will be calculated from $-\partial V / \partial r_{o}$, as above. Now,

$$
\begin{aligned}
V\left(r_{o}\right)= & -2 G \sigma\left[\int_{0}^{a} \mathrm{~d} y \int_{0}^{\sqrt{a^{2}-y^{2}}} \frac{\mathrm{d} x}{\sqrt{\left(r_{o}-y\right)^{2}+x^{2}}}\right. \\
& \left.+\int_{0}^{a} \mathrm{~d} y \int_{0}^{\sqrt{a^{2}-y^{2}}} \frac{\mathrm{d} x}{\sqrt{\left(r_{o}+y\right)^{2}+x^{2}}}\right]
\end{aligned}
$$

The first term represents the contribution from the right half of the disk in Figure 6, while the second represents the contribution from the left half of the disk. After calculating $-\partial V / \partial r$ and performing a variety of tedious algebraic manipulations, we obtain, using the relative coordinates $r_{a}$ and $y_{a}$, the expression for the radial component of the force, $F\left(r_{a}\right)$ :

$$
\begin{aligned}
& F\left(r_{a}<1\right)=2 G \sigma\left[\int_{0}^{1} \frac{\mathrm{d} y_{a}\left(r_{a}-y_{a}\right)}{\left(\sqrt{1-y_{a}^{2}}+\sqrt{1+r_{a}^{2}-2 y_{a} r_{a}}\right) \sqrt{1+r_{a}^{2}-2 y_{a} r_{a}}}\right. \\
& \left.+\int_{0}^{1} \frac{\mathrm{d} y_{a}\left(r_{a}+y_{a}\right)}{\left(\sqrt{1-y_{a}^{2}}+\sqrt{1+r_{a}^{2}+2 y_{a} r_{a}}\right) \sqrt{1+r_{a}^{2}+2 y_{a} r_{a}}}+\ln \left(\left(1-r_{a}\right) /\left(1+r_{a}\right)\right)\right]
\end{aligned}
$$




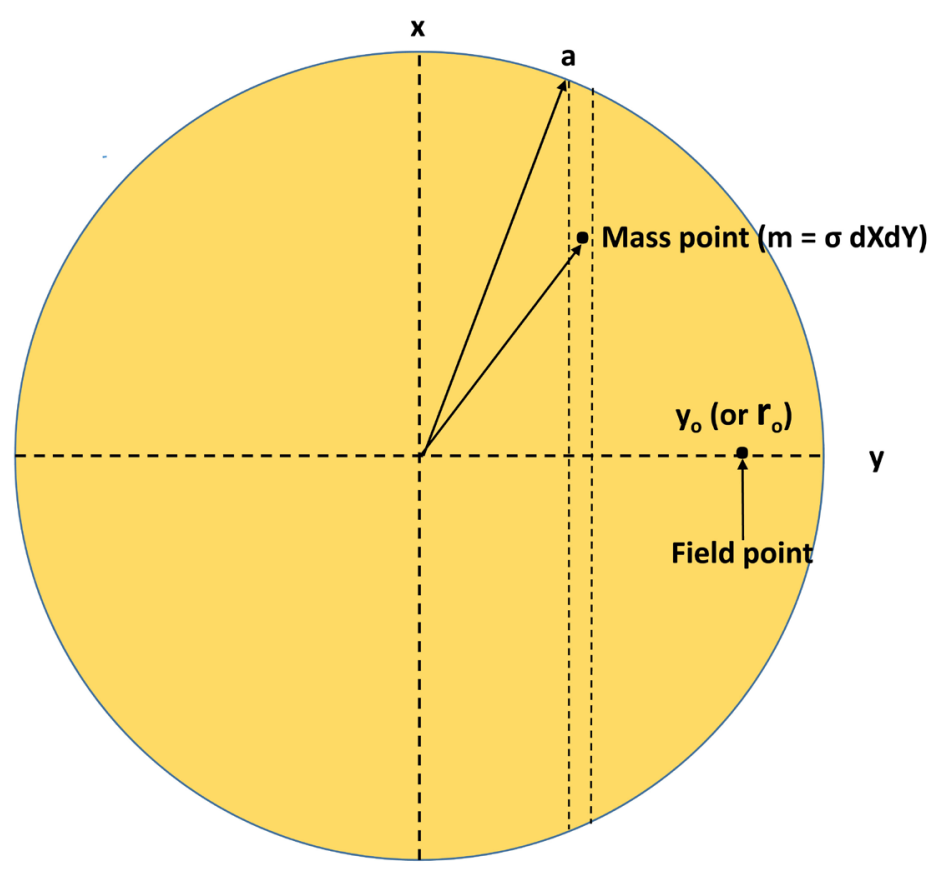

Figure 6. The geometry needed for the use of numerical integration, as a method of calculating the radial force in the plane of the disk. In this case, the field point coordinate is within the disk $\left(y_{o}<a\right)$, but it could be beyond the disk $\left(y_{o}>a\right)$.

For $r_{a}>1$, the expression for the force is the same, except that $\left(1-r_{a}\right)$ within the logarithm is replaced by $\left(r_{a}-1\right)$. We note that the logarithmic divergence appears explicitly in the expression for the force at $r_{a}=1$, as opposed to the expression in Equation (3). The "standard" numerical integration is used to calculate numerical value of the two integrals in Equation (5) over the range of $r_{a}$. This procedure is available online in [6]. In Figure 7 are three curves representing the radial force. Two of them indicate that Equations (3) and (5) produce indistinguishable results, as they should. The third, based on a particular sum of Legendre polynomials, produces agreement with the other two methods, except near $r_{a}=1$, which we shall now examine.

\section{Legendre Polynomials}

Figure 8 shows the azimuthally symmetric disk geometry needed for the use of Legendre polynomials of varying degree $l, P_{l}(\cos \theta)$. The gravitational potential, $V(r, \theta)$, is expressed, for this geometry, as:

$$
\begin{gathered}
V(r, \theta)=\sum_{0}^{\infty} A_{l} r^{l} P_{l}(\cos \theta) ; r<a \\
V(r, \theta)=\sum_{0}^{\infty} B_{l} r^{-(l+1)} P_{l}(\cos \theta) ; r>a
\end{gathered}
$$

In the case of other azimuthally symmetric geometries, such as the annular region between a disk and a ring, the potential could be expressed as the sum of these two equations. These expressions are standard for solutions of Laplace's Equation with azimuthal symmetry and can be found in any textbook on the subject. 


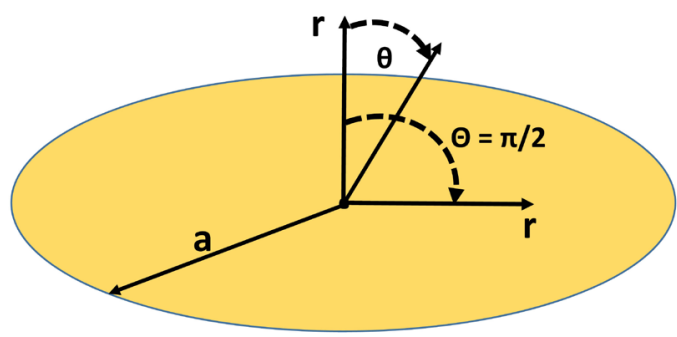

Figure 7. Geometry for the application of the Legendre polynomials to the disk problem, where " $a$ " and " $r$ " are simply lengths and $\theta$ is the angle with respect to the normal to the disk.

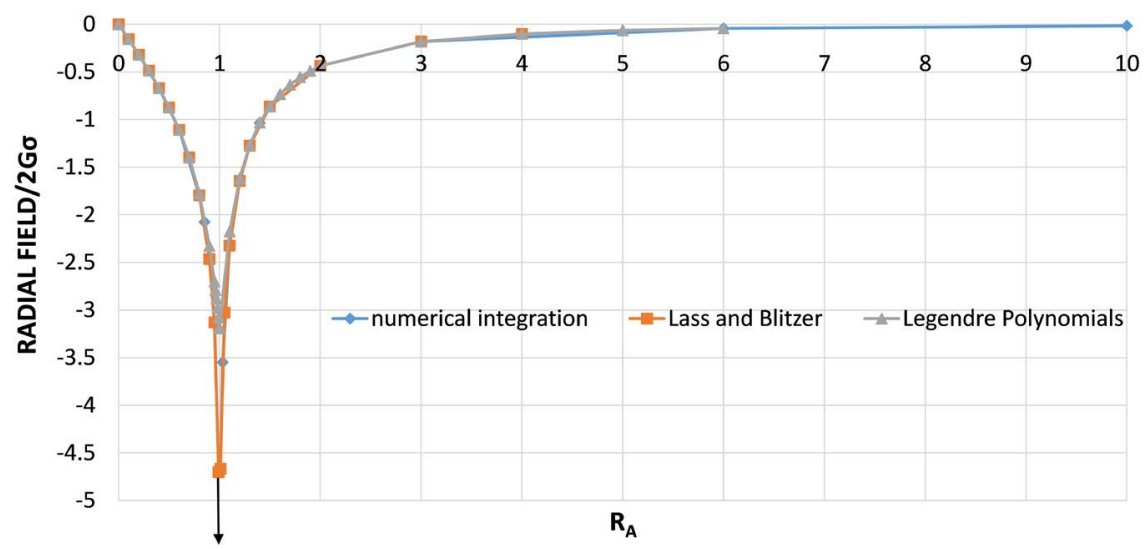

Figure 8. Gravitational field vs. distance from center, using the three different calculational methods identified in the legend. All three agree very well, except very near $r_{a}=1$, where the logarithmic divergence occurs and the use of Legendre polynomials falls short.

Since $P(1)=1$ for all 1 , we first determine $V(r, \theta)$ on-axis, where $\theta=0$, as in Figure 8, and expand the potential in powers of $(r / a)$ for $r<a$, and in powers of $(a / r)$ for $r>a$. We then multiply each term in either series by $P_{(}(\cos \theta)$ and sum over the chosen number of products in the resultant series. In our case, where the potential is in the plane of the disk, $\theta=\pi / 2$, and all terms involve even $I=2 n$. As stated in [8], another website for "Mathematics Stack Exchange", $P_{2 n}(\cos \theta)=P_{2 n}(0)=(-1)^{n}(2 n) ! / 2^{2 n}(n !)^{2}$. All odd terms, $P_{2 n+1}(0)$, are zero. The on-axis potential, $V(r, 0)$ is:

$$
V(r, 0)=-(2 G \sigma) \pi\left[\sqrt{r^{2}+a^{2}}-r\right]
$$

For $r<a$, one factors out the a-term under the square root, which allows for the expansion of the potential in powers of $(r / a)$. Factoring out the r-term allows for the expansion in powers of $(a / r)$. After performing the expansions out to a respectable six terms (the chosen number), making use of the expressions for $P_{2 n}(0)$, and calculating the derivative $-\partial V / \partial r$, we obtain the following for $F_{r}$ : (using the relative coordinate $r_{a}$ )

$$
\begin{aligned}
F_{r}= & -(2 G \sigma) \pi\left[0.5 r_{a}+0.1875 r_{a}^{3}+(0.1171 \cdots) r_{a}^{5}+(0.0854 \cdots) r_{a}^{7}\right. \\
& \left.+(0.06729 \cdots) r_{a}^{9}+(0.0555 \cdots) r_{a}^{11}\right] ; r_{a}<1
\end{aligned}
$$




$$
\begin{aligned}
F_{r}= & -(2 G \sigma) \pi\left[\frac{0.5}{r_{a}^{2}}+\frac{0.1875}{r_{a}^{4}}+\frac{(0.1171 \cdots)}{r_{a}^{6}}+\frac{(0.0854 \cdots)}{r_{a}^{8}}\right. \\
& \left.+\frac{(0.06729 \cdots)}{r_{a}^{10}}+\frac{(0.0593 \cdots)}{r_{a}^{12}}\right] ; r_{a}>1
\end{aligned}
$$

Although it is difficult to resolve details, Figure 8 shows how well the use of Legendre polynomials agrees with the other two methods, except near $r_{a}=1$. An expanded view of this region is shown in Figure 9, where we can see the disagreement developing between $0.8<r_{a}<1.2$. As the curve of $F_{r}$ vs $r_{a}$ grows steeper in its approach, from either direction, to the logarithmic divergence at $r_{a}=1$, more terms in the Legendre expansion are needed to accurately describe it. An even more respectable eight terms in the expansion would shrink the range of disagreement to $0.9<r_{a}<1.1$. As a motivation for their paper, Lass and Blitzer [2] make a general comment concerning the inconveniently large number of terms required for an accurate Legendre expansion, but no specifics are given.

\section{Z-Dependence of the Radial Field and the Field in the Z-Direction}

So far, we have only looked at the radial field in the plane of the disk, but what about the radial field at different values of $z_{a}$ and the vertical field at different values of $r_{a}$ ? Both of these fields can be readily calculated using the "standard" numerical integration, as in Equation (4), but with the inclusion of $z_{o}$ in the expression for distance, as in Equation (9). The potential gradient, $-\partial V / \partial r$, is then calculated.

$$
\begin{aligned}
V\left(r_{o}, z_{o}\right)= & -2 G \sigma\left[\int_{0}^{a} \mathrm{~d} y \int_{0}^{\sqrt{a^{2}-y^{2}}} \frac{\mathrm{d} x}{\sqrt{\left(r_{o}-y\right)^{2}+z_{o}^{2}+x^{2}}}\right. \\
& \left.+\int_{0}^{a} \mathrm{~d} y \int_{0}^{\sqrt{a^{2}-y^{2}}} \frac{\mathrm{d} x}{\sqrt{\left(r_{o}+y\right)^{2}+z_{o}^{2}+x^{2}}}\right]
\end{aligned}
$$

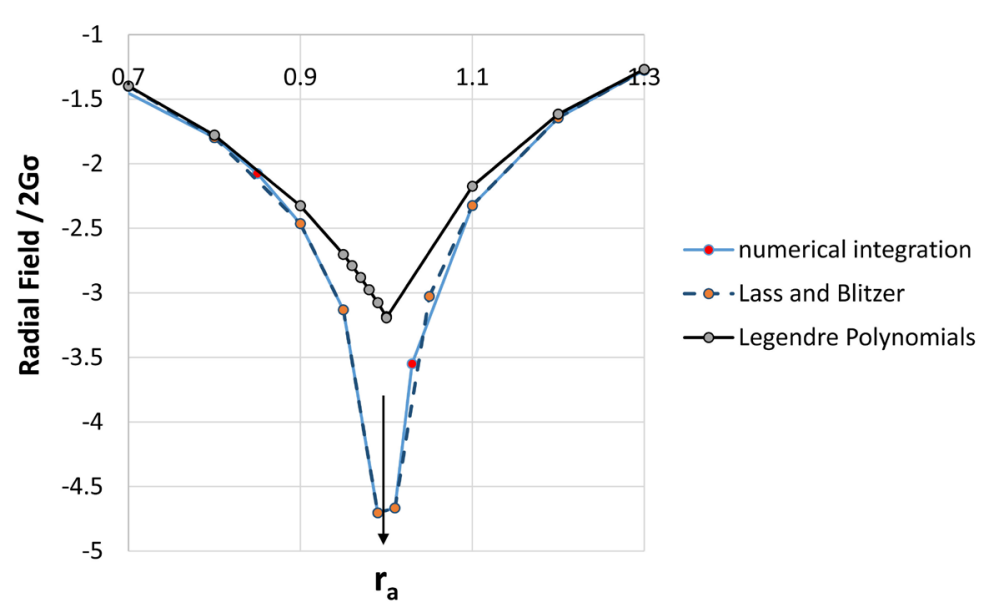

Figure 9. An expanded version of Figure 8, demonstrating more clearly where the use of Legendre polynomials deviates from Lass and Blitzer and numerical integration. 
Figure 10 shows the first of these results for $z_{a}=0,0.2$, and 0.4 . The basic shapes are the same for all three curves, but there is no logarithmic divergence for values of $z_{a}>0$ because there is no edge to the mass distribution at those levels. As with the first curve, these two curves do peak at $r_{a}=1$ because at that point, all of the mass exerts a force in the same direction; there is no cancellation in the radial direction. Although that is also true for $r_{a}>1$, the distance to $r_{a}$ has increased to all of the mass points on the disk.

Figure 11 shows the field in the z-direction, $F_{z}(=-\partial V / \partial z)$, for various values of $r_{a}$, both less than and greater than 1 . For all $r_{a}<1, F_{z}=-2 \pi \sigma G$ at $z=0$. This

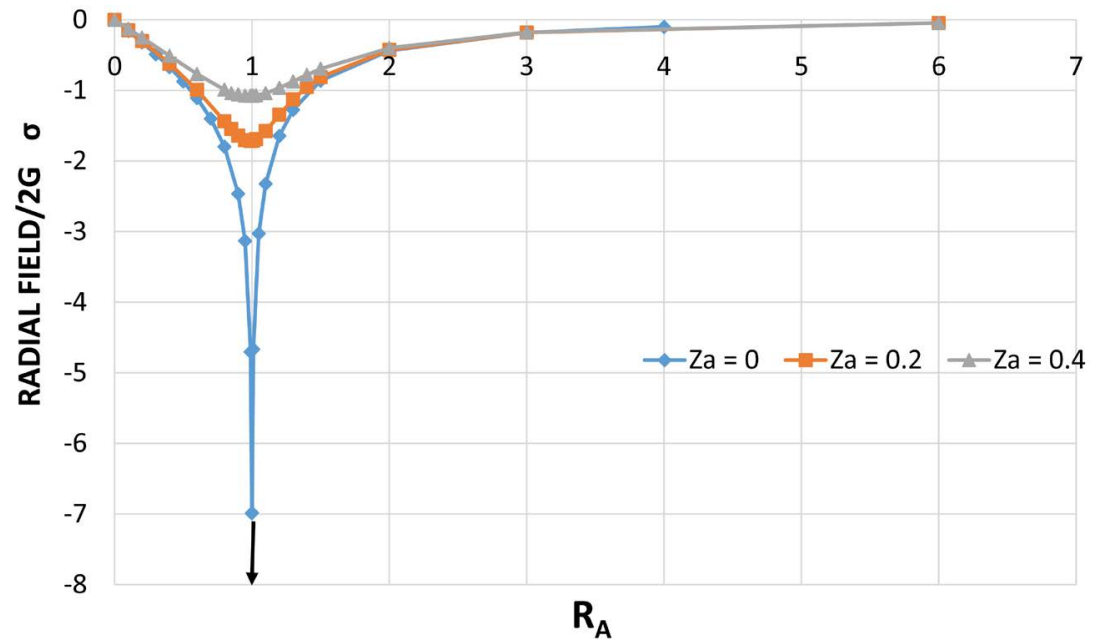

Figure 10. Radial gravitational field at three values of $z_{a}$, using "standard" numerical integration.

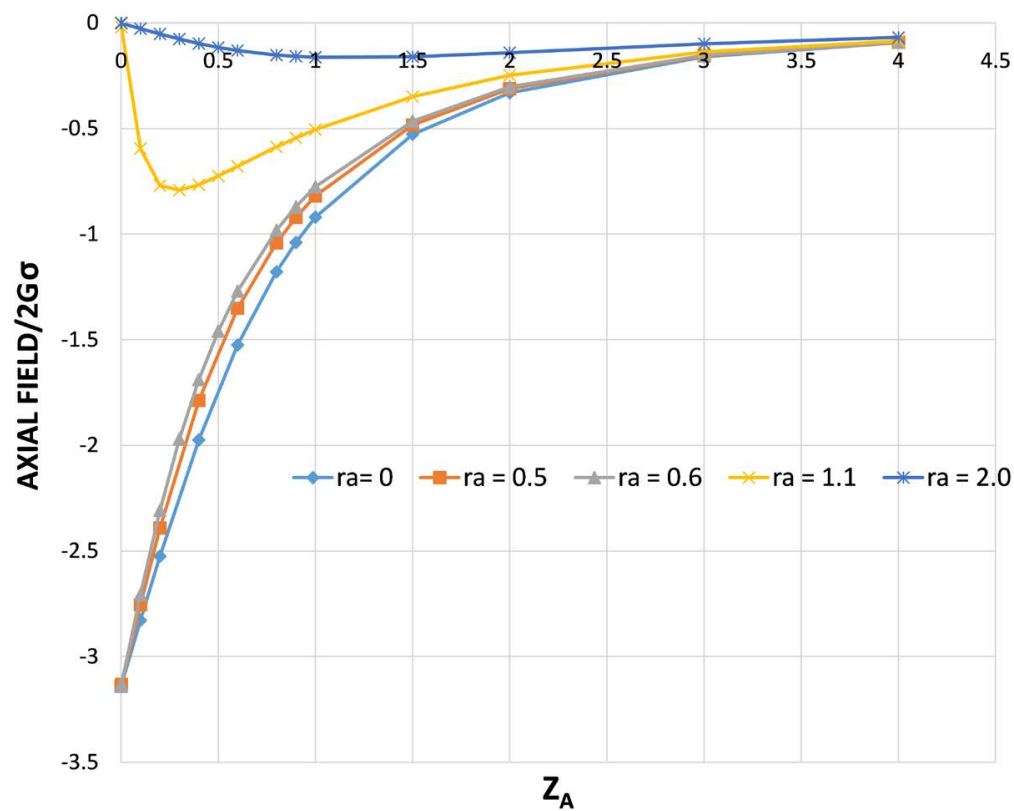

Figure 11. Gravitational field in z-direction for solid disk for various values of $r_{a}$ out to 4 . Three are for $r_{a}<1$ and two for $r_{a}>1$. Note that the first three peak at $r_{a}=0$ and the remaining are zero at $r_{a}=0$. 
result follows from the Gaussian formulation of the law of gravitation, $\nabla \boldsymbol{F}=$ $-4 \pi G \rho$, where $F$ is the gravitational field and $\rho$ is the mass density at each point. Using a Gaussian pillbox, as in electrostatics, that straddles the surface mass density of the disk, along with the integral form of Gauss' law, leads to the result. All mass points on the disk, other than the one in question, only contribute to the field parallel to the surface of the disk at $z=0$.

We note that $F_{z}=0$ for all $r_{a}>1$ and $z=0$, where there is no surface mass ( $\sigma=$ 0 ). We could also note that by planar anti-symmetry, the field above the disk points in the negative $z$-direction, while that below points in the positive $z$-direction. Since there is no discontinuity in the field for $r_{a}>1$ and $z_{a}=0$, what else could the field be for those values of $r_{a}$ and $z_{a}$ ? Apropos of these comments, Figure 12 illustrates the $F_{z}$ in the plane of a disk with a hole in it. Within the entire area of hole and the region beyond the disk, $F_{z}=0$ in the plane of the disk.

This is definitely not true for the radial field, as we now discuss in the case of a solid disk containing a hole of half of its area and centered within it. The radial field can readily be obtained from linear superposition by subtracting the field of a smaller disk, identical in size and position of the hole, from that of the solid disk. Figure 13 shows the fields of the two disks, while Figure 14 shows the result of the subtraction. The counter-intuitive result is that radial field within the hole is in the positive radial direction and increases to the edge of the hole, where the logarithmic divergence occurs. It continues in that direction, with reduced magnitude, into bulk of the remaining mass of the original disk, until $r_{a} \approx$ 0.81 . At that point, the field becomes negative and stays that way for all $r_{a}>0.81$, eventually approaching zero for large $r_{a}$.

By comparison, Figure 15 shows the gravitational field, $F_{s}$ of a sphere of mass density $\rho$, radius a, and a central hole of half the volume of the sphere. Because of its spherical symmetry, this is a simple problem, also solved with linear superposition, whose solution is:

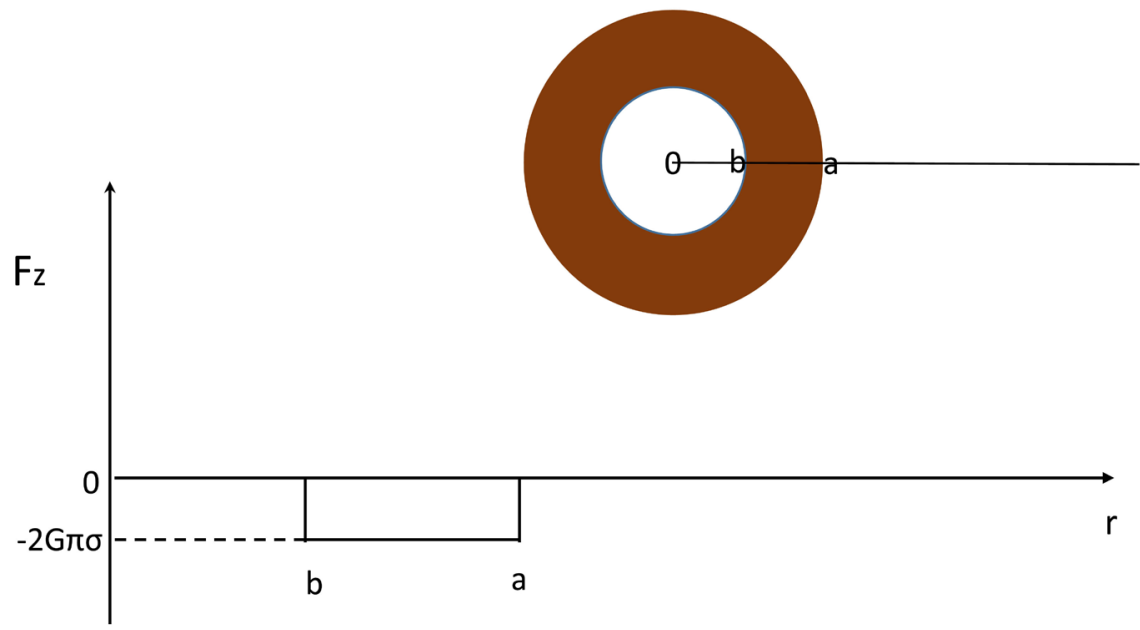

Figure 12. $F_{z}$ (gravitational field in $z$-direction) as a function of radius of a disk of radius " $a$ " with a hole of radius " $b$ ". In the plane of the disk. 


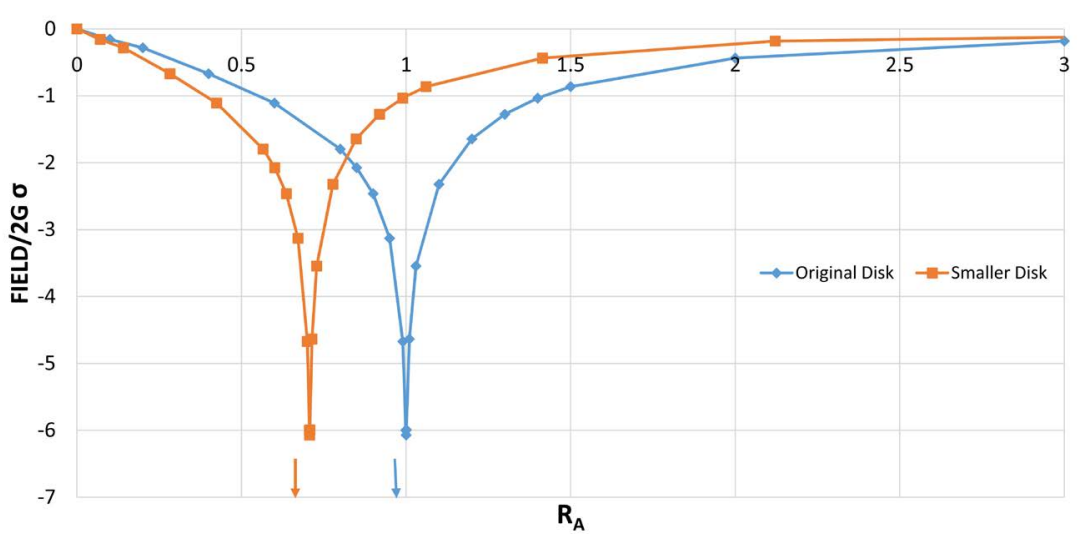

Figure 13. The radial field from the two disks. The smaller disk is the size of the hole that will be created by subtraction.

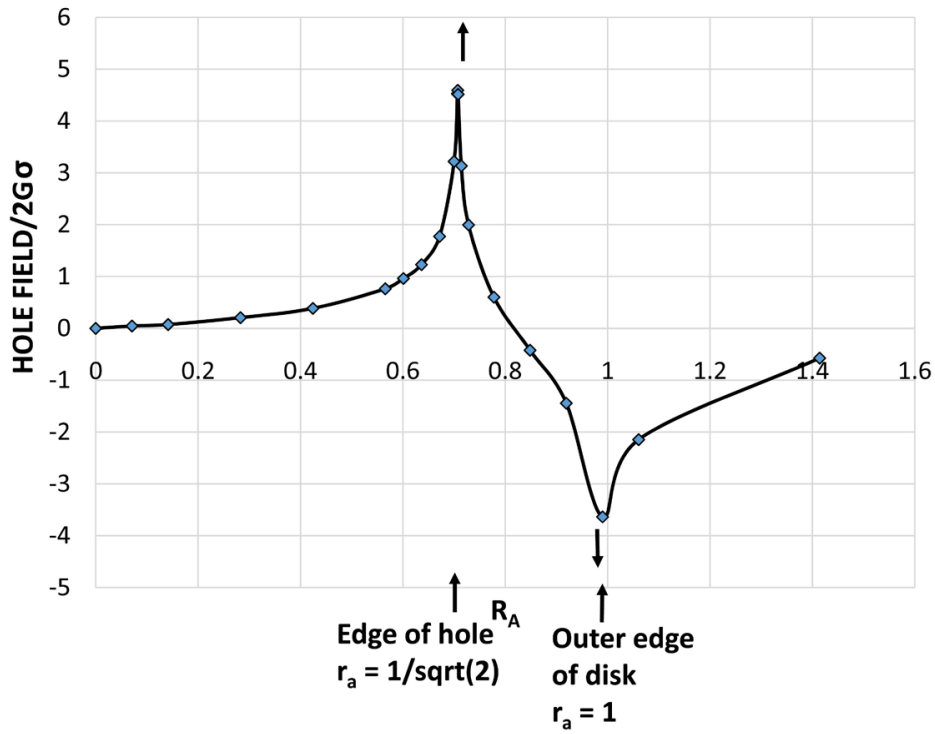

Figure 14. Gravitational field in the hole, in the remaining mass of the disk, and somewhat beyond the mass of the disk.

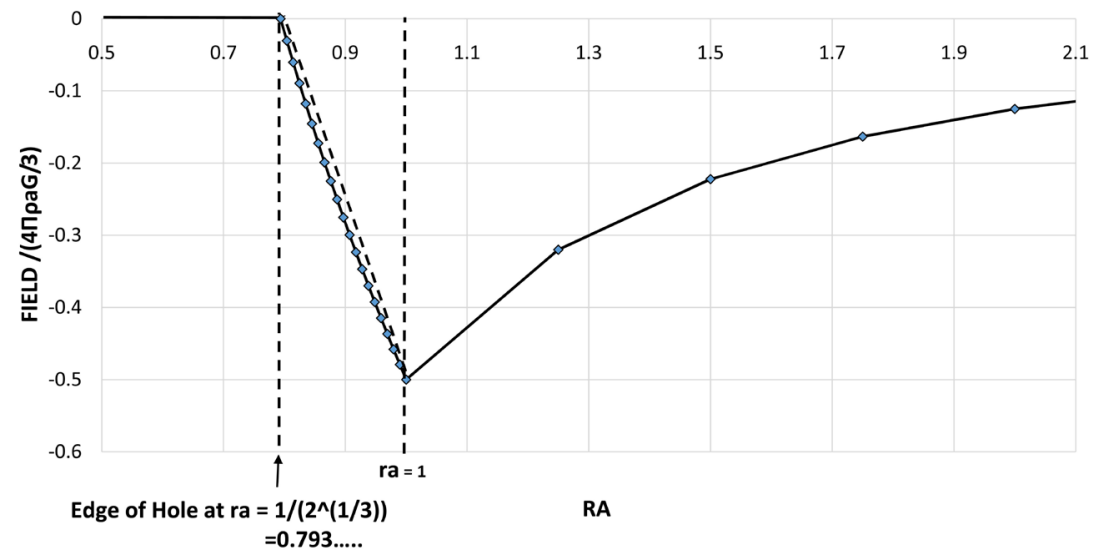

Figure 15. Gravitational field of a sphere with a central hole that removes half the mass of the solid sphere. The diagonal dotted line shows the slight deviation from linearity for $r_{a}<$ 1 . 


$$
\begin{gathered}
F_{s}=\left(\frac{4 \pi}{3}\right) G a \rho\left[r_{a}-\frac{1}{2 r_{a}^{2}}\right] ; 0.5^{1 / 3} \leq r_{a} \leq 1 \\
F_{s}=\left(\frac{2 \pi}{3}\right) \frac{[G a \rho]}{r_{a}^{2}} ; r_{a} \geq 1
\end{gathered}
$$

$F_{s}=0$ within the hole, where $0 \leq r_{a} \leq 0.5^{1 / 3}$.

\section{Disk of Non-Zero Thickness}

Until now, we have concentrated on a disk of zero thickness. In this section, we consider the radial gravitational field of a uniform disk of non-zero thickness $T$ equal to a maximum of one tenth of its fixed radius " $a$ ". Two different situations are considered, as shown in Figure 16. In situation A, the field point $\mathrm{X}$ is fixed at the lowest corner, while the relative thickness of the disk can change from 0 to $T_{a}$ $=0.1$. The field at $\mathrm{X}$ is determined as a function of the relative thickness, $Z_{a}$ $\left(\leq T_{a}\right)$. In situation $\mathrm{B}$, the relative thickness is a constant $T_{a}$, but the field point $\mathrm{X}$ moves from the lower corner along the outer surface of the disk to the top corner. The field at $\mathrm{X}$ is determined as a function of its relative position, $z_{a}$. There are two contributions to the field in situation B: the section of the disk above $z_{a}$ and the one below. For a disk of constant radius with respect to height, these contributions will have already been determined in situation A.

These contributions are determined by breaking up the disk into thin slices and considering each slice to be a disk of zero thickness, having a vertical separation from point $\mathrm{X}$. The contribution from a given slice is calculated as in Equation (9) and taking its radial gradient at $r_{a}=1$. The total contribution is obtained by integrating the individual contributions over the relative thickness, $Z_{a}$. Figure 17 illustrates the geometry.

Figure 18 shows the contribution to the field at $\mathrm{X}$ as a function of $z_{a}$. The function is obviously linear in $\ln \left(z_{a}\right)$ over the range of consideration. The curve would deviate significantly from linearity for much larger $Z_{a}$ because the

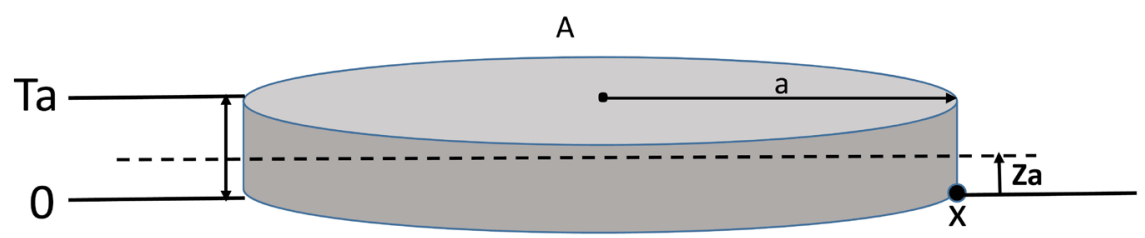

B

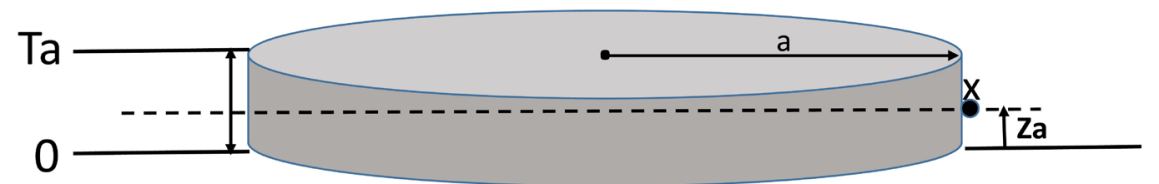

Figure 16. Basic geometry for the gravitational field of a disk of maximum relative thickness $T_{a}$, radius a, and constant density $\rho$. In A, the field point $\mathrm{X}$ is fixed at the lower corner, with the thickness of the disk varying along relative coordinate $z_{a}$ from 0 to $T_{a}$. In $\mathrm{B}$, the thickness of the disk is fixed at $T_{a}$ and the position of $\mathrm{X}$ varies from 0 to $T_{a}$. 


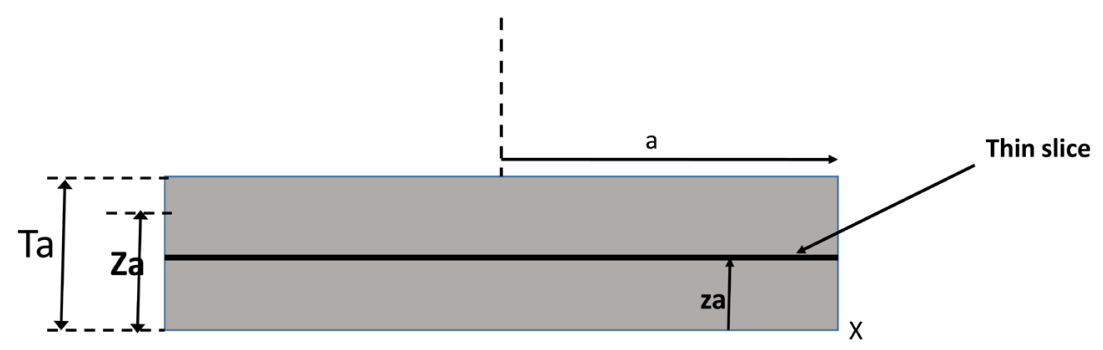

Figure 17. Vertical cross-section of the disk, showing relevant characteristics.

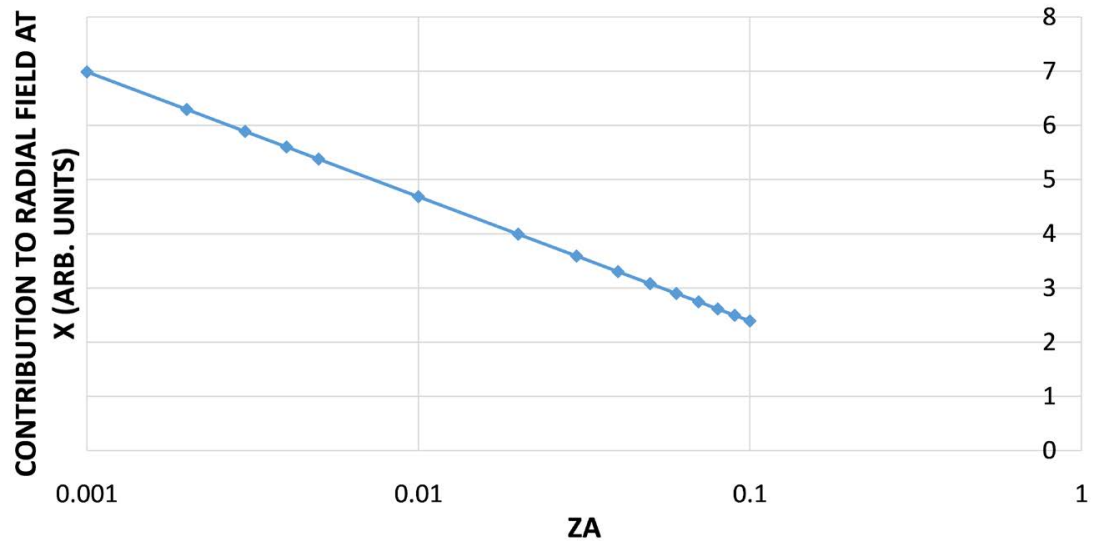

Figure 18. The contribution to the radial field at $\mathrm{X}$ from a slice at $Z_{\text {a }}$.

contribution must asymptotically approach zero for very thick disks. Calling the function $F\left(z_{a}\right)$,

$$
F\left(z_{a}\right)=-\ln \left(z_{a}\right)+0.0869
$$

To obtain the total contribution at $\mathrm{X}$ for situation $\mathrm{A}$, these contributions must be summed from 0 to $Z_{a}$. Thus, the total contribution over that thickness is:

$$
\int_{0}^{Z_{a}} F\left(Z_{a}\right) \mathrm{d} Z_{a}=-Z_{a} \ln \left(Z_{a}\right)+1.0869 Z_{a}
$$

Aside from a scale factor, this equation is plotted in Figure 19 as A. Graph B is obtained by adding the contributions from the entire regions above and below $\mathrm{X}$, as obtained from $\mathrm{A}$. The symmetry about $z_{a}=0.05$ is obvious, as are values at the end points, both of which receive contributions from the entire disk.

\section{Properties of Circular Orbits in the Plane of a Disk}

The properties of these circular orbits considered are the orbital period as a function of their radius measured from the center of the disk and the mass of the disk. The disk is assumed to be an approximate representation of the Milky Way with the same mass and diameter. The estimated diameter of the Milky Way is $10^{5} \mathrm{ly}$ [9], among others, and the estimated mass is $1.4 \times 10^{42} \mathrm{~kg}$ [10]. Since the mean thickness of the Milky Way is estimated to be only about $(1-2) \times 10^{3}$ ly [9], it will be ignored. In addition, the stability of such orbits with respect to radial perturbations and perturbations perpendicular to the plane of the disk are considered. 


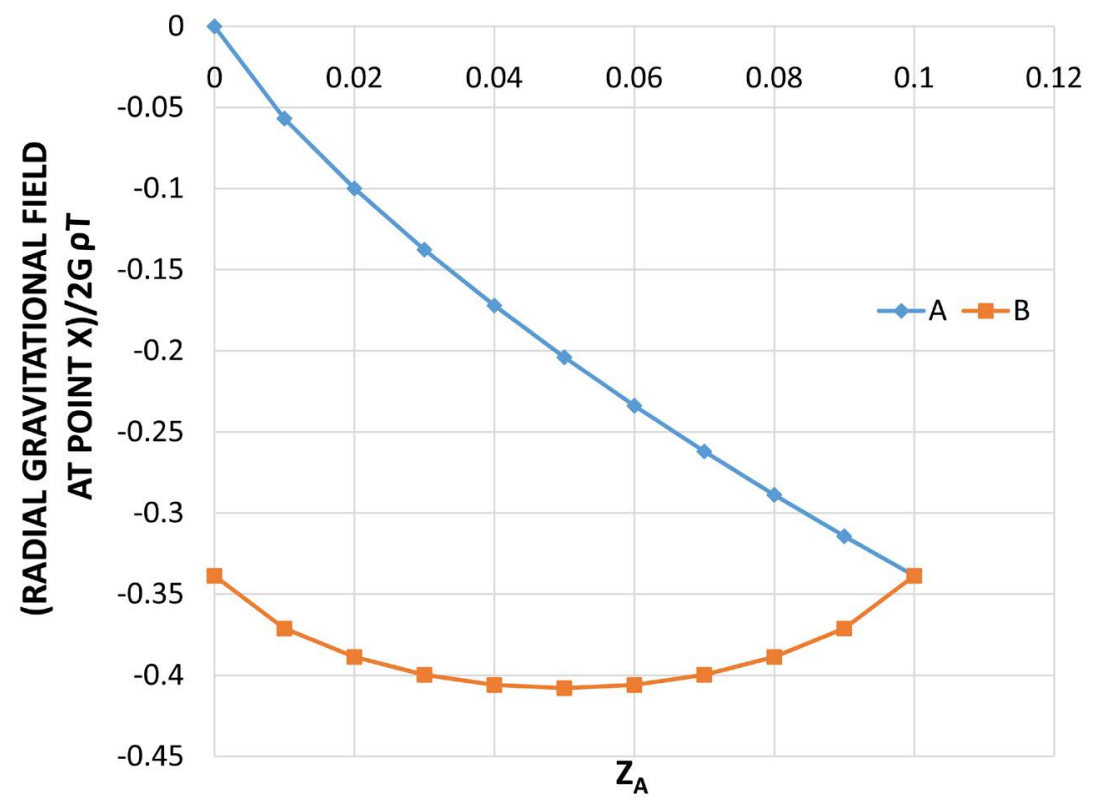

Figure 19. The radial gravitational field at point $\mathrm{X}$ for $\mathrm{A}$ and $\mathrm{B}$.

Finding the period of the orbit is a simple problem in orbital mechanics. Assuming the radial field, $F(R)$, beyond the disk is as in Figure 2, but including $2 G \sigma$, then

$$
F(R)=\frac{V^{2}}{R},
$$

where $V$ and $R$ are the orbital speed and radius, respectively. The period $T$ is

$$
T=\frac{2 \pi R}{V}=2 \pi \sqrt{R / F}
$$

Figure 20 shows the orbital period in billions of years plotted against the orbital radius in light years, up to $5 \times 10^{5} \mathrm{ly}$. Galaxies that are less than this distance from the Milky Way are listed in [11], but no information allowing for a comparison to Figure 20, if any exists, has been found by this author. It is of some interest, however, to determine at what orbital radius the orbital period deviates negligibly from that of a point mass of the same mass as the disk. A simple calculation shows that the radius is about $75 \times 10^{3} \mathrm{ly}$, or 1.5 galactic radii, which suggests a rapid march toward the asymptotic limit (but not as rapid as in the case of a sphere!). The result for a disk was arrived at by noting that the period of the orbit of radius $R$ around a point mass is, coincidentally, proportional to $R^{1.5}$. Thus, if Figure 20 were displayed on a log-log plot, its behavior would become essentially linear starting at about 1.5 galactic radii. Figure 21 is such a plot showing the transition to linearity, with a slope of about 1.5 , as indicated by the accompanying (eyeballed) dashed line.

Regarding orbital stability perpendicular to the plane of the disk, we can see in Figure 11 that the force in the positive $z$-direction for radii beyond the disk is negative. By anti-symmetry, the opposite is also true. Thus, the force out of the plane of the disk is always restorative and, therefore, maintains vertical stability 


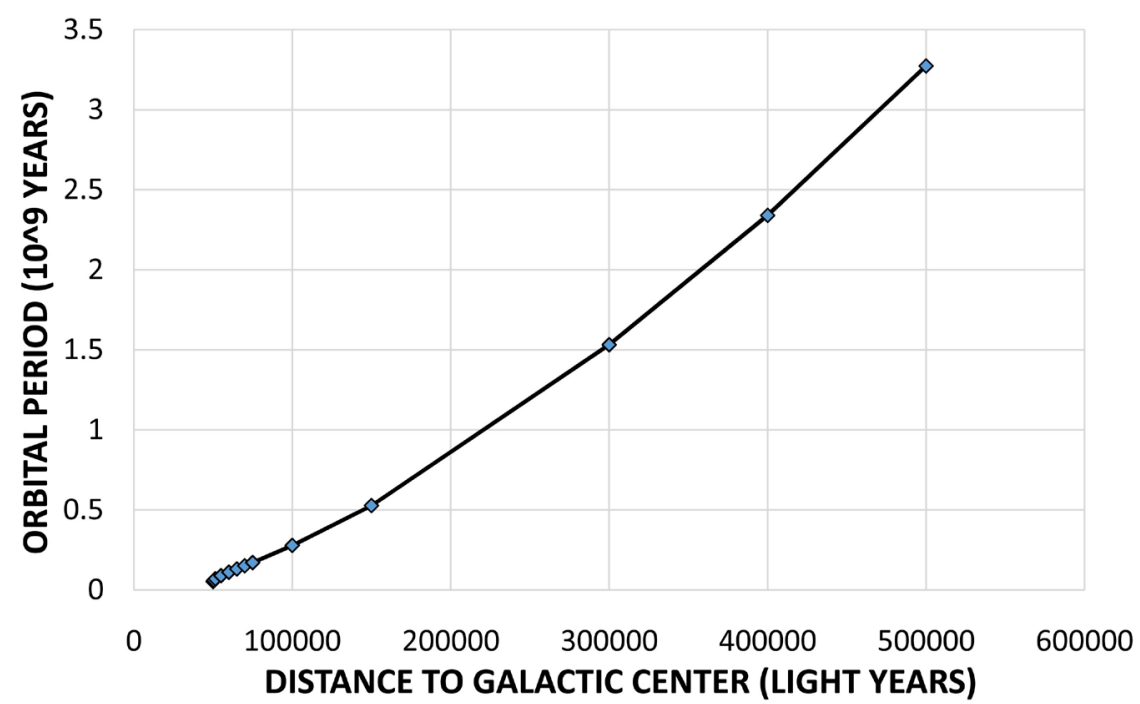

Figure 20. Orbital period of a satellite galaxy around the Milky Way, assuming a circular orbit in the plane of the Milky Way. Assumed radius and mass of Milky Way = 50,000 ly and $6 \times 10^{42} \mathrm{~kg}$, respectively.

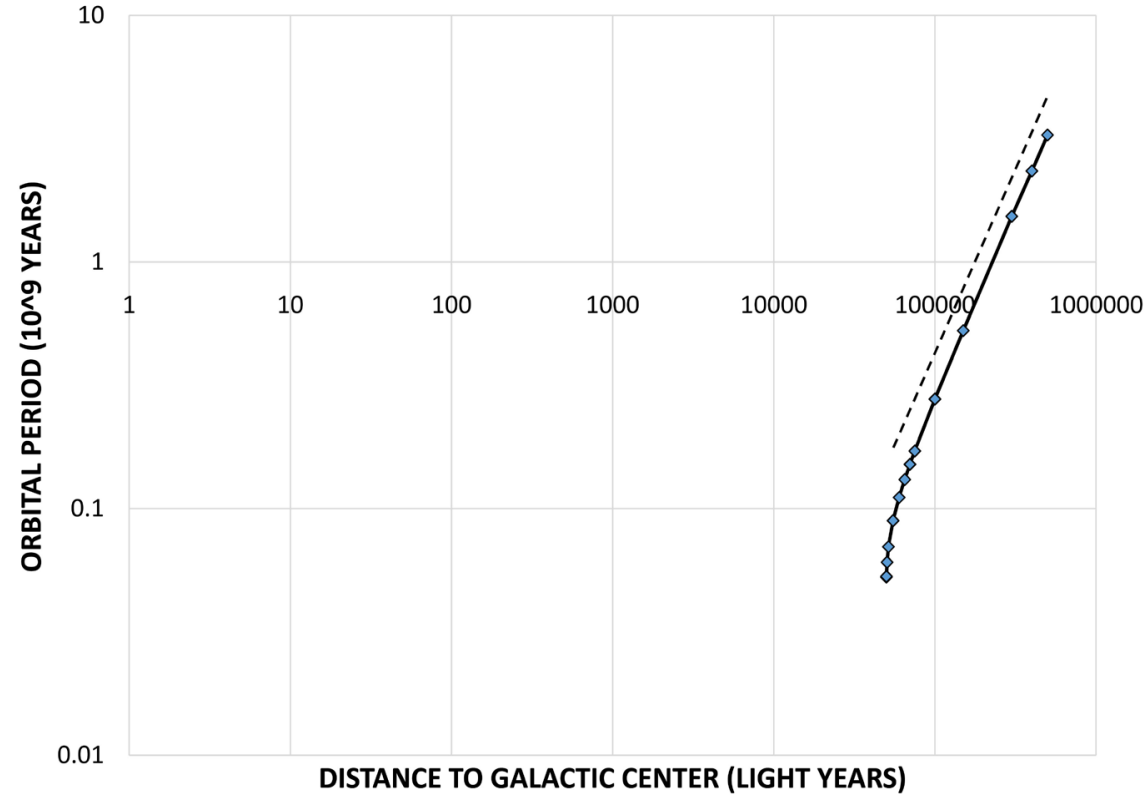

Figure 21. Log-log version of Figure 20, showing the distance at which the disk effectively behaves as a point mass, as indicated by the dashed line.

(i.e., a vertical oscillation about the plane of the disk). One could imagine a horizontal orbit half way between two identical disks, but such an orbit would be vertically unstable, since a perturbation toward either disk would only create a growing force imbalance toward that disk. This is particularly true for $r_{a}<1$, where the magnitude of the negative force is monotonically decreasing with $z_{a}$. The orbiting object would eventually collide with the disk. This trend would not necessarily hold for $r_{a}>1$, such as $r_{a}=1.1$, where the magnitude of the negative force increases with $z_{a}$ for $0 \leq z_{a} \leq 0.3$. 
Radial stability of a circular orbit is discussed in [12], which is a physics lecture. It occurs within the context of a coordinate system rotating with the orbiting particle, and thus includes the fictitious outward centrifugal force along with the real inward gravitational force. These two forces balance in the absence of a radial perturbation, thereby maintaining the orbital radius constant. In the presence of a perturbation, the author derives the condition for which an outward perturbation in the radius will induce a negative radial force, and vice versa, thereby causing an oscillation about the equilibrium radius. The condition for stability is:

$$
\frac{3}{\rho}+\frac{g^{\prime}(\rho)}{g(\rho)}>0
$$

where $\rho$ is the radius of the unperturbed circle, $g(\rho)$ is the negative of the gravitational force, and $g^{\prime}(\rho)$ is its derivative at $\rho$. Figure 22 is a plot of the above expression, called "stability condition", for the field of a thin disk, as in Figure 7. Since extreme accuracy was not required for the current purpose of displaying the range of stability, the derivative was approximated by differences between successive calculated points. We see that radial stability occurs only for $r_{a} \gtrsim 1.15$. If the field behaved according to an inverse-square law, as outside a sphere, stability would occur for all values of $r_{a}$.

\section{The Escape Velocity of the Earth from the Milky Way}

In this section, we shall calculate the escape velocity from the disk-approximation of the Milky Way based solely on the gravitational attraction to an escaping object as a function of its location within the galaxy. The object's orbital motion, or

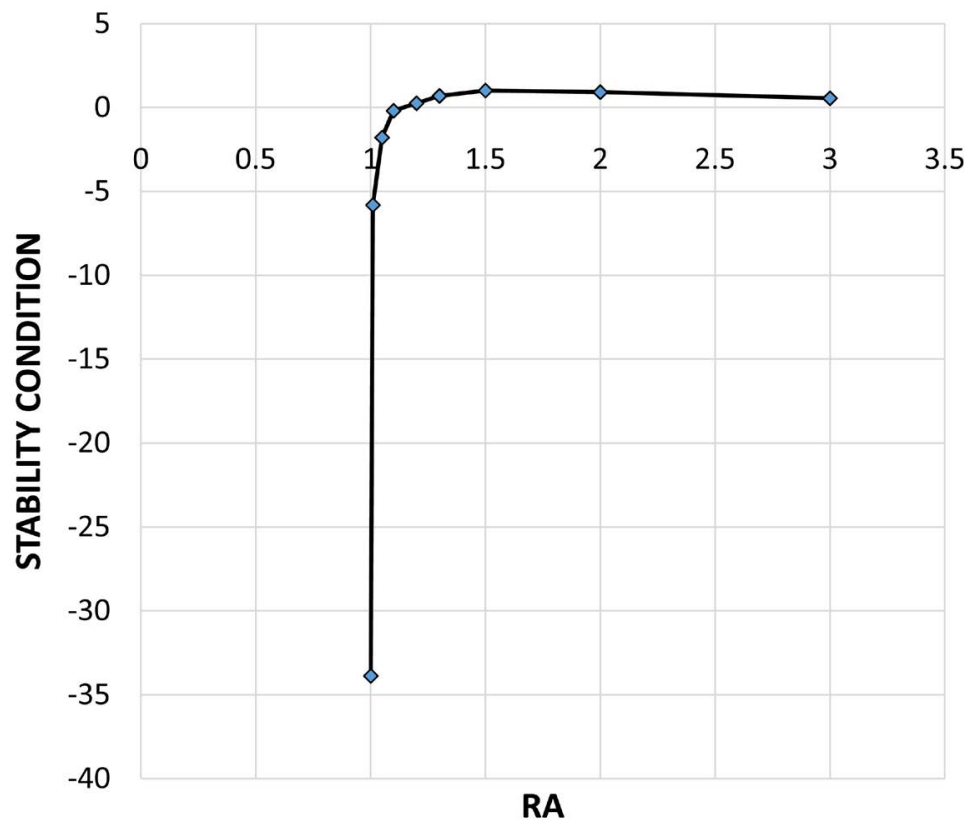

Figure 22. Stability condition for a particle in orbit around a disk and in the plane of the disk. 
other motion, within the galaxy, which influences the escape velocity, is not considered. As is well known, the escape velocity or, more properly, the escape speed, $V_{e s o}$ is computed by equating the object's initial kinetic energy to the negative of its initial potential energy. Thus,

$$
v_{\text {esc }}=\sqrt{2 V\left(r_{a}\right)}
$$

where $V\left(r_{a}\right)$ is the potential energy, as in Equation(4), using a relative coordinate. This expression gives the escape velocity as a function of position within the disk. Figure 23 is a graph of the computed escape velocity vs the radial position of the object. Since our solar system is $\approx 26,000$ ly [9] from the center of the Milky Way, $r_{a}$ for it $\approx 0.5$, where its computed escape velocity from Equation (16) is $684 \mathrm{~km} / \mathrm{sec}$. A reported value is $\approx 550 \mathrm{~km} / \mathrm{sec}$ [9]. The $24 \%$ discrepancy could be considered fortuitously small, in view of the uncertainties of the various estimates in the literature and the use of the disk approximation in this paper.

\section{Summary}

In this paper, we have presented certain aspects of the gravitational characteristics of the disk geometry, including an ideally thin disk, a disk of finite thickness, and a thin disk in the form of a ring, though not thin walled. This geometry has been less studied than that of a sphere, which, because of its higher degree of symmetry, is simpler to analyze. Aside from its interest simply as a physics problem, certain astronomical objects, such as the Milky Way and protoplanetary disks, are disk-like in shape. In that regard, we have also presented calculations of the circular orbits in the plane of the Milky Way, approximated as a disk, concerning their orbital period and orbital stability. The escape velocity of an object, particularly at the position of the solar system, has been similarly calculated.

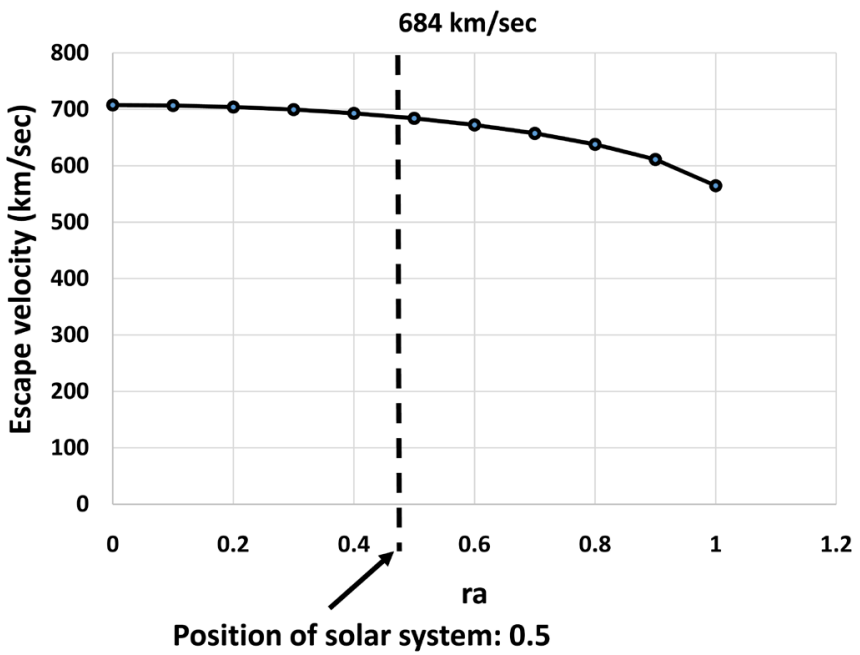

Figure 23. Escape velocity from Milky Way, assuming that the galactic radius $=50,000$ ly, the galactic mass $=1.42 \times 10^{42} \mathrm{~kg}$, and the position of the solar system $\approx 0.5$ galactic radii from the center. Reported escape velocity $=550 \mathrm{~km} / \mathrm{sec}$. The effect of the orbital velocity of the sun around the galactic center is not included. 


\section{Acknowledgements}

The author thanks W. F. Filter, retired Sandia National Laboratories physicist, for reviewing the draft of this paper and making many valuable suggestions for its improvement.

\section{Conflicts of Interest}

The author of this paper declares that he has no conflict of interest of any sort, particularly as described by the journal under "potential conflict of interest."

\section{References}

[1] https://en.wikipedia.org/wiki/Protoplanetary disk

[2] Lass, H. and Blitzer, L. (1983) The Gravitational Potential Due to Uniform Disks and Rings. Celestial Mechanics, 30, 225-228.

http://adsbit.harvard.edu//full/1983CeMec..30..225L/0000225.000.html https://doi.org/10.1007/BF01232189

[3] Krough, F.T., Ng, E.W. and Snyder, W.V. (1982) The Gravitational Field of a Disk. Celestial Mechanics, 26, 395-405.

http://adsbit.harvard.edu//full/1982CeMec..26..395K/0000395.000.html https://doi.org/10.1007/BF01230419

[4] "Elliptic Integral" in Wikipedia. https://en.wikipedia.org/wiki/Elliptic integral

[5] Keisan Online Calculator. https://keisan.casio.com/exec/system/1180573451

[6] Integral Calculator. https://www.integral-calculator.com

[7] Mathematics Stack Exchange.

https://math.stackexchange.com/questions/1111135/asymptotic-expansion-of-the-c omplete-elliptic-integral-of-the-first-kind/2737375

[8] Mathematics Stack Exchange.

https://math.stackexchange.com/questions/1805794/prove-p-2n0-1n-frac2n22nn2of-legendre-polynomials

[9] "Milky Way" in Wikipedia. https://en.wikipedia.org/wiki/Milky Way

[10] "Measuring the Milky Way: One Massive Problem, One New Solution". https://phys.org/news/2016-05-milky-massive-problem-solution.html

[11] “List of Nearest Galaxies" in Wikipedia. https://en.wikipedia.org/wiki/Listofnearestgalaxies

[12] Dzierba, A.R. Circular Orbits and Their Stability: A Physics Lecture. http://www.dzre.com/alex/P441/lectures/lec 22.pdf 